\title{
A Step-Wise Procedure to Develop an Algorithm for Image Brightness Using Edge Detection Principles
}

\author{
A.Prema ${ }^{1}$, Prof.Dr.M.V.RamanaMurthy ${ }^{2}$ \\ (1*)Research Scholar, Department of Computer Science, Karpagam University, Coimbatore, Tamilnadu, India, \\ (2) Chairman and Head, Department of Computer Science and Mathematics, Osmania University, Hyderabad, \\ India,
}

\begin{abstract}
This paper uses the technique of edge detection to make the image of an oil pipeline toappear bright and clear for further investigations after applying Mathematical morphology for curve error detection. This algorithm is developed to read the pixel values and eliminate the shadow images that appear. The pixels are studied carefully to give a set of appropriate values, which helps in setting up the right image for image evaluation. To achieve this, edge detection method is used and an algorithm is developed for image processing. This algorithm helps to build up a set of accurate pixel values, to determine the co-ordinates of the image for processing and analysis.This algorithm is developed mainly to remove the shadow images if caused during the camera intervention inside the oil pipeline.
\end{abstract}

Keywords: Edge, Smooth, Noise, Thresold, Gradient

Why edge detection?

\section{Introduction}

Edge:The line or part where an object or area begins or ends.

An edge detection has to be carried out to know the actual area or length of a pipeline under analysis. After series of evaluation and careful study, Mathematical morphology was applied to detect the crack defects in the images taken from an oil pipeline. At certain situations it developed to show shadow images. Though it is avoidable, it is necessary to develop an algorithm to rectify this problem, if not this will be a big ladder down strike in developing this research paper.

Image shading mainly occurs at the point wherever the pipeline is smooth and deep, because of the oil transportation. This occurs due to the yearlong usage of the pipeline, for carrying the oil.

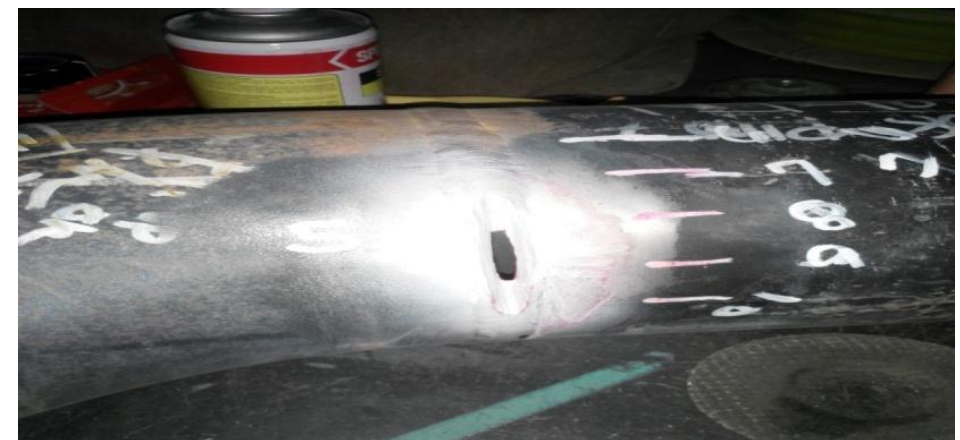

Figure 1:A pipeline showing Crack with measurement (picture courtesy-SADARA,KSA)

\section{Approaches}

Edge detection has many methods, but they are grouped in to two categories

1.Search based

2.Zero Crossing based

The edge detection methods that are used mainly differ in the types of smoothing filters used and the way measures of edge strength are computed.

The different types of edge detection algorithms are:

- Gradient based algorithm

- Laplacian based algorithm

- Canny's edge detection algorithm

Canny's edge detection algorithm performs better than all the algorithms.Before starting with the process,few definitions has to be known and they are listed below: 


\section{Hysteresis}

Hysteresis is the dependence of a system not only on its current environment but also on its past environment. This dependence arises because the system can be in more than one internal state. To predict its future development, either its internal state or its history must be known

\section{What is Smoothing of an image?}

In image processing, to smooth a data set is to create an approximating function that attempts to capture important patterns in the data, while leaving out noise or other fine-scale structures or rapid phenomena. In smoothing, the data points of a signal are modified so individual points (presumably because of noise) are reduced, and points that are lower than the adjacent points are increased leading to a smoother signal.

Smoothing may be used in two important ways that can aid in data analysis

(1) by being able to extract more information from the data as long as the assumption of smoothing is reasonable, and

(2) by being able to provide analyses that are both flexible and robust. Image Noise

Image noise is a random (not present in the object imaged) variation of brightness or color information in images, and is usually an aspect of electronic noise. It can be produced by the sensor and circuitry of a scanner ordigital camera.

\section{Canny's Edge Detection Algorithm}

The Canny edge detection algorithm is known as the optimal edge detector.

In this technique, a list of criteria is followed to improve current methods of edge detection.

The first and most obvious is low error rate. It is important that edges occurring in images should not be missed and that there be NO responses to non-edges.

The second criterion is that the edge points be well localized. In other words, the distance between the edge pixels as found by the detector and the actual edge is to be at a minimum.

A third criterion is to have only one response to a single edge.

This was implemented because the first 2 were not substantial enough to completely eliminate the possibility of multiple responses to an edge.

Based on these criteria, the canny edge detector first smoothes the image to eliminate and noise.

It then finds the image gradient to highlight regions with high spatial derivatives. The algorithm then tracks along these regions and suppresses any pixel that is not at the maximum. The gradient array is now further reduced by hysteresis.

Hysteresis is used to track along the remaining pixels that have not been suppressed. Hysteresis uses two thresholds and if the magnitude is below the first threshold, it is set to zero (made a nonedge). If the magnitude is above the high threshold, it is made an edge. And if the magnitude is between the 2 thresholds, then it is set to zero unless there is a path from this pixel to a pixel with a gradient above T2.

\section{Step1}

In order to implement the canny edge detector algorithm, a series of steps must be followed. The first step is to filter out any noise in the original image before trying to locate and detect any edges. And because the Gaussian filter can be computed using a simple mask, it is used exclusively in the Canny algorithm. Once a suitable mask has been calculated, the Gaussian smoothing can be performed using standard convolution methods. A convolution mask is usually much smaller than the actual image. As a result, the mask is slid over the image, manipulating a square of pixels at a time. The larger the width of the Gaussian mask, the lower is the detector's sensitivity to noise. The localization error in the detected edges also increases slightly as the Gaussian width is increased. The Gaussian mask used in my implementation is shown below. 


\begin{tabular}{|c|c|c|c|c|}
\hline 2 & 4 & 5 & 4 & 2 \\
\hline 4 & 9 & 12 & 9 & 4 \\
\hline 5 & 12 & 15 & 12 & 5 \\
\hline 4 & 9 & 12 & 9 & 4 \\
\hline 2 & 4 & 5 & 4 & 2 \\
\hline
\end{tabular}

Step2

Figure 3 Discrete approximation to Gaussian function with $\sigma=1.4$

After smoothing the image and eliminating the noise, the next step is to find the edge strength by taking the gradient of the image. The Sobel operator performs a 2-D spatial gradient measurement on an image. Then, the approximate absolute gradient magnitude (edge strength) at each point can be found. The Sobel operator uses a pair of $3 \times 3$ convolution masks, one estimating the gradient in the $\mathrm{x}$-direction (columns) and the other estimating the gradient in the $y$-direction (rows). They are shown below:

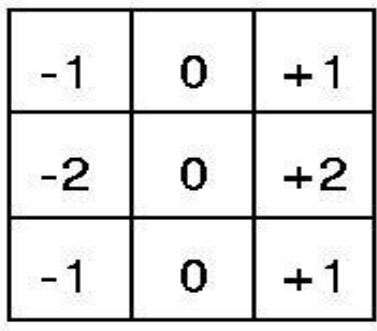

Gx

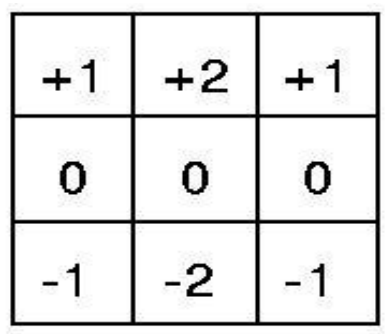

Gy

The magnitude, or edge strength, of the gradient is then approximated using the formula:

$|\mathrm{G}|=|\mathrm{Gx}|+|\mathrm{Gy}|$

\section{Step 3}

The direction of the edge is computed using the gradient in the $\mathrm{x}$ and $\mathrm{y}$ directions. However, an error will be generated when sumX is equal to zero. So in the code there has to be a restriction set whenever this takes place. Whenever the gradient in the $\mathrm{x}$ direction is equal to zero, the edge direction has to be equal to 90 degrees or 0 degrees, depending on what the value of the gradient in the y-direction is equal to. If GY has a value of zero, the edge direction will equal 0 degrees. Otherwise the edge direction will equal 90 degrees. The formula for finding the edge direction is just:

Theta $=$ invtan $(\mathrm{Gy} / \mathrm{Gx})$

$\underline{\text { Step4 }}$

Once the edge direction is known, the next step is to relate the edge direction to a direction that can be traced in an image. So if the pixels of a $5 \times 5$ image are aligned as follows:

\begin{tabular}{|c|c|c|c|}
\hline $\mathrm{X}$ & $\mathrm{X}$ & $\mathrm{X}$ & $\mathrm{X}$ \\
\hline $\mathrm{X}$ & $\mathrm{X}$ & $\mathrm{X}$ & $\mathrm{X}$ \\
\hline$X$ & $\mathrm{X}$ & $\mathrm{a}$ & $\mathrm{X}$ \\
\hline $\mathrm{X}$ & $\mathrm{X}$ & $\mathrm{X}$ & $\mathrm{X}$ \\
\hline
\end{tabular}

$\mathrm{x} \quad \mathrm{x} \quad \mathrm{x} \quad \mathrm{x}$
Then, it can be seen by looking at pixel " $\mathrm{a}$ ", there are only four possible directions when describing the surrounding pixels - $\mathbf{0}$ degrees (in the horizontal direction), 45 degrees (along the positive diagonal), 90 degrees (in the vertical direction), or 135 degrees (along the negative diagonal). So now the edge orientation has to be resolved into one of these four directions depending on which direction it is closest to (e.g. if the orientation angle is found to be 3 degrees, make it zero degrees). Think of this as taking a semicircle and dividing it into 5 regions. 


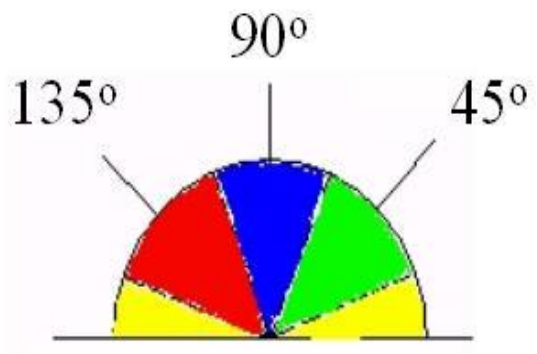

Therefore, any edge direction falling within the

( 0 to $22.5 \& 157.5$ to 180 degrees) is set to 0 degrees. Any edge direction falling in the green range (22.5 to 67.5 degrees) is set to 45 degrees. Any edge direction falling in the blue range (67.5 to 112.5 degrees) is set to 90 degrees. And finally, any edge direction falling within the red range (112.5 to 157.5 degrees) is set to 135 degrees.

$\underline{\text { Step } 5}$

After the edge directions are known, non-maximum suppression now has to be applied. Nonmaximum suppression is used to trace along the edge in the edge direction and suppress any pixel value (sets it equal to 0 ) that is not considered to be an edge. This will give a thin line in the output image.

Step 6

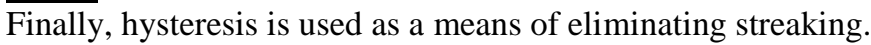

Streaking is the breaking up of an edge contour caused by the operator output fluctuating above and below the threshold. If a single threshold, T1 is applied to an image, and an edge has an average strength equal to T1, then due to noise, there will be instances where the edge dips below the threshold. Equally it will also extend above the threshold making an edge look like a dashed line. To avoid this, hysteresis uses 2 thresholds, a high and a low. Any pixel in the image that has a value greater than T1 is presumed to be an edge pixel, and is marked as such immediately. Then, any pixels that are connected to this edge pixel and that have a value greater than T2 are also selected as edge pixels.If an edge has to be followed a gradient $\mathrm{T} 2$ is required to start and to end $\mathrm{T} 1$ is required.

\section{Conclusion}

After applying all these 6 steps,image clarity for edge detection is achieved.Steps has to be followed one after the other to remove shadows and to detect the starting and end point of the image that is considered for pattern analysis.Since Digital camera is used to take the pictures inside the pipeline,Noise as expected is reduced using this procedure(Edge-Detection).This can be further extended to know the middle pixel range to improve the quality of the picture to carry out a smooth pattern recognition.

\section{References}

[1]. Edge detection with Sobel operator by Sausheong,April 20,2011

[2]. A Research on Improved Canny Edge Detection Algorithm-Jun Li, Sheng Ding 